\title{
The Tully-Fisher Relation for Hickson Compact Groups
}

\author{
Philippe Amram ${ }^{1}$, Claudia Mendes de Oliveira ${ }^{2}$, Henri Plana ${ }^{3}$, and \\ Chantal Balkowski ${ }^{4}$ \\ ${ }^{1}$ Observatoire de Marseille, France; ${ }^{2}$ Instituto de Astronomia, \\ São Paulo, Brazil; ${ }^{3}$ Universidade de Santa Cruz, Brazil; ${ }^{4}$ Observatoire \\ de Paris, France
}

\begin{abstract}
We investigate the properties of the $B$-band Tully-Fisher (TF) relation for 25 compact group galaxies, using $V_{\max }$ derived from 2 -D velocity maps. Our main result is that the majority of the Hickson Compact Group (HCG) galaxies lie on the TF relation, although with large scatter. However, $20 \%$ of the galaxies, including the lowest-mass systems, seem to have higher $B$ luminosities, for a given mass, or alternatively, a mass which is too low for their luminosities. We favour the scenario of brightening of the outliers due to either enhanced star formation or merging, rather than truncation of the dark halo due to interactions, to explain the position of the outliers on the $\mathrm{TF}$ relation.
\end{abstract}

The data used in this paper are gathered from three publications (Mendes de Oliveira et al. 1998, ApJ, 507, 691; Plana et al. 2003, AJ, 125, 1736; Amram et al. 2003, A\&A, 402, 865) which studied the kinematics of galaxies in 9 compact groups (HCG 10, 16, 19, 87, 88, 89, 91, 96, 100). Our main result is that galaxies in compact groups follow the TF relation, with the exception of a few of the lowest mass members. This result is in contrast with an earlier result by Rubin et al. (1991, ApJS, 76, 153) who found an offset of the TF relation for most galaxies in compact groups in the sense that galaxies in compact groups have lower velocities for a given luminosity. Generally, a choice of parameters guided by photometry alone (when no kinematic maps are available) will result in a lower $\mathrm{V}_{\max }$ of a galaxy, affecting the $\mathrm{TF}$ relation in the direction of the Rubin et al. result. It is clear from Figure 1 that a few of the lowest-mass members of compact groups seem to lie off the relationship. If they once had the TF parameters similar to galaxies in a less dense environment, they could have either brightened by 1 to 2 magnitudes in the blue band, to get to their present position, or they could have lost a substantial amount of mass, or a combination of both. A natural way that the smaller members could have brightened, especially in a hostile environment like in compact groups, is by forming stars, induced by the eventual interactions the galaxy may have suffered within the group. A second mechanism to leave the outliers in the position where they are in the TF relation would be by mass loss, within $R_{25}$. This could be caused, for example, by disruption or ablation of the dark halo due to strong interactions in the group. The fact that the $B$-band Tully-Fisher relation is similar for compact group and field galaxies tells us that these galaxies show common mass-to-size relations and that the halos of compact group galaxies have not been significantly stripped inside $R_{25}$. In general, rotation curves of compact group galaxies are 


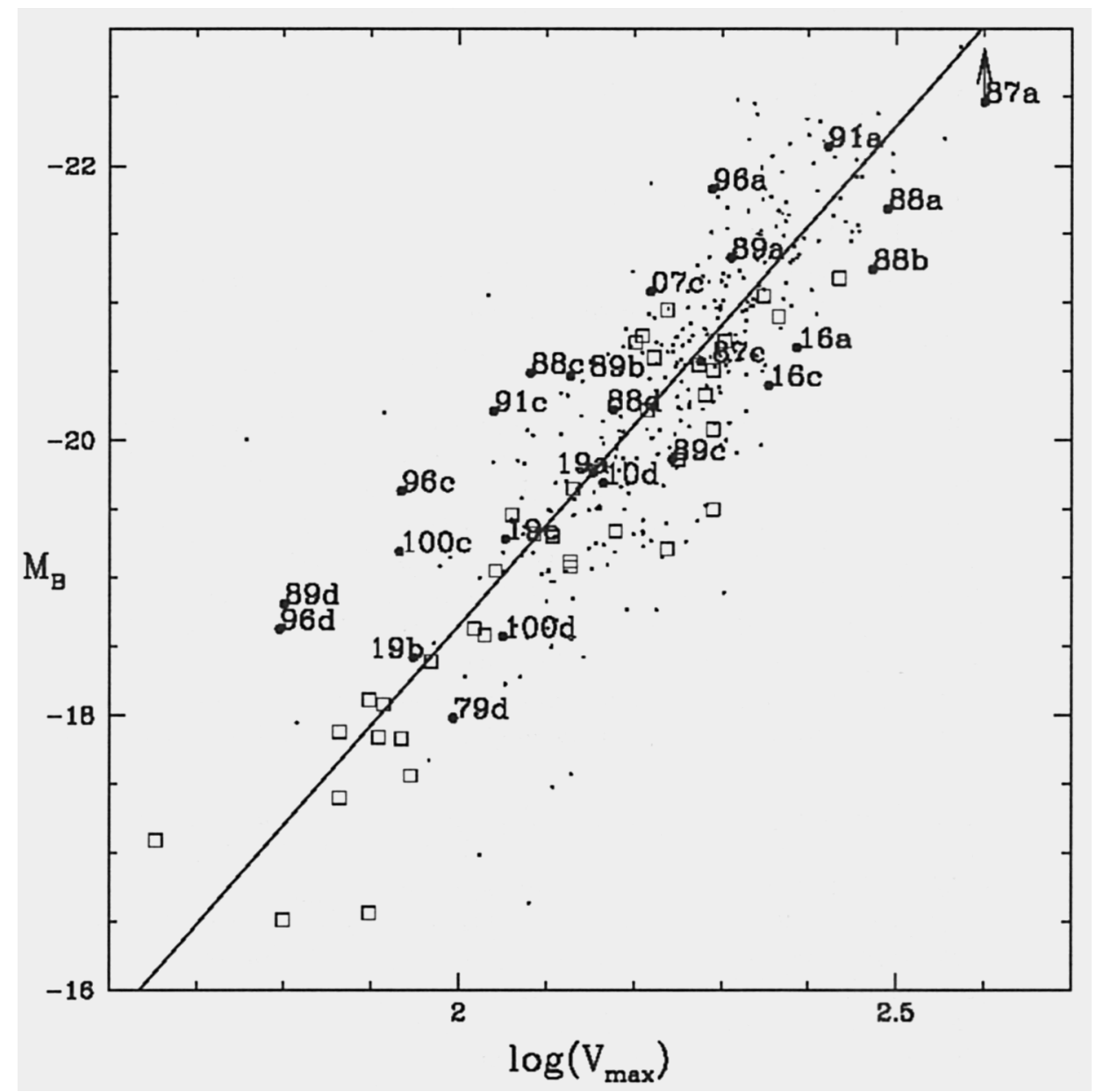

Figure 1. The Tully Fisher relation for samples of compact group (black dots), field (small dots) and Ursa Major cluster galaxies (squares). The solid line represents the fiducial TF relation for local calibrators with known distances from Cepheids (Tully \& Pierce 2000, ApJ, 533, 744). Galaxies HCG 79d and HCG 87a are known to be dusty and therefore their magnitudes are probably brighter than what is plotted here.

well behaved in the sense that the two sides of the curves approximately match and they are either flat or rising in the last measured points. However, two of the HCG galaxies which are the most massive members in M51-like pairs, HCG 91a and HCG 96a, have very asymmetric rotation curves, with one arm rising and the other one falling, indicating most probably a recent perturbation by the small close companions. A more complete analysis and discussion of the TullyFisher relation for Hickson Compact Groups is given in Mendes de Oliveira, Amram, Plana \& Balkowski (2003, AJ, in press). 